\section{Depression and mortality in a high-risk population}

\author{
II-Year follow-up of the Medical Research Council Elderly \\ Hypertension Trial
}

MELANIE ABAS, MATTHEW HOTOPF and MARTIN PRINCE

\begin{abstract}
Background It is not clear whether the increased mortality associated with depression can be explained by the effects of potential confounding variables.
\end{abstract}

\begin{abstract}
Aims To measure the effect of depression on mortality after controlling for cognitive decline, cardiovascular risk factors and antidepressant use.
\end{abstract}

Method A prospective cohort study derived from data from a multi-centre randomised controlled trial of moderate hypertension. A total of 2584 participants, aged 65-75 years at study entry, were followed up for II years.

\section{Results Depression on the}

SelfCARE-D scale was associated with mortality after controlling for gender. After controlling for cardiovascular risk factors, cognitive decline and antidepressant use, depression continued to have a modest effect (hazard ratio $=1.43$; 95\% Cl I.03-1.98). Depression in males and in people aged under 70 years significantly increased the risk of death.

\section{Conclusions Depression was}

associated with mortality only after controlling for gender. There was a modest but robust association between depression and mortality that was not explained by confounding by cardiovascular risk factors, cognitive decline or history of antidepressant use.

\section{Declaration of interest Funded by} the UK Medical Research Council. M.A. has received an educational award from Eli Lilly Ltd.
Despite fairly consistent evidence for the association between depression and mortality (Wulsin et al, 1999; Saz \& Dewey, 2001), a number of questions remain. Several conventional cardiovascular risk factors (e.g. smoking) are associated with psychiatric disorder (Ismail et al, 2000). Unless these are controlled for, it is hard to know whether the increased mortality simply reflects the lifestyle profiles of these populations. Cognitive decline and dementia are strongly associated with mortality, and depression is frequently an early sign of cognitive decline in elderly populations (Witthaus et al, 1999). It is clearly important to control for all these confounders before postulating depression as an independent risk factor for mortality. Furthermore, new papers have suggested that the effect of depression on mortality may be greater in males than females and in major as opposed to minor depression (Penninx et al, 1999; Schoevers et al, 2000; Saz \& Dewey, 2001).

\section{METHOD}

\section{Study setting and design}

The Medical Research Council Elderly Hypertension Trial was a randomised, placebo-controlled, single-blind trial of the treatment of moderate hypertension (mean systolic blood pressure $=160-209 \mathrm{mmHg}$, mean diastolic blood pressure $<115 \mathrm{mmHg}$ ) in 4396 subjects aged 65-74 years, conducted from 1983 to 1989 in 226 general practices across the UK (MRC Working Party, 1992). Exclusion criteria were known serious cardiovascular or cerebrovascular disease, cancer or dementia. Subjects were tagged via their National Health Service numbers. The date of death, if this occurred, was collected until the end of follow-up in September 1994. Thus, 11 years of follow-up data were available.

Data on a range of variables were collected at baseline. Some variables were also measured at the 1-, 9-, 21- and 54-month follow-up assessments. Patients were randomised to three groups: beta-blocker (atenolol, $50 \mathrm{mg}$ daily), thiazide diuretic (hydrochlorothiazide, $25 \mathrm{mg}$ or $50 \mathrm{mg}$, plus amiloride, 2.5 or $5 \mathrm{mg}$ daily) or placebo. The results of the main study have been published elsewhere (MRC Working Party, 1992). In summary, both treatments reduced blood pressure compared with placebo. Furthermore, the diuretic group, but not the beta-blocker group, had significantly reduced risks of stroke, coronary events and all cardiovascular events, compared with placebo.

\section{Participants}

The subjects for the current paper were those who took part in the psychiatric substudy, namely the first 2680 recruits for the main study. Of these 2680,29 refused to participate. Of the 2651 who agreed, $2584(97.5 \%)$ contributed adequate data on the main explanatory measures. These constitute the current sample.

\section{Measures}

\section{Depression status}

Depression was measured on a validated 12-item depression scale, the SelfCARE-D. This is a self-administered depression scale derived from the Comprehensive Assessment and Referral Evaluation depression scale and was developed specifically for use in elderly subjects in the primary care setting (Bird et al, 1987). Each of the 12 questions are scored either 0 or 1 , therefore scores are in the range $0-12$. In the present study, the SelfCARE-D was applied at entry and at each subsequent follow-up. Bird et al (1987) suggested that a cut-off of $\geqslant 6$ out of 12 is indicative of clinical depression in the general elderly population. However, a subsequent study using the Geriatric Mental State (GMS; Copeland et al, 1986) as the gold standard found that the mean score for cases of depression was $\geqslant 7$, with a specificity of only $45 \%$ for the $\geqslant 6$ cut-off (Blanchard et al, 1994). Another study carried out in elderly people with physical illness recommended a cutoff of $\geqslant 7$ (Banerjee et al, 1998). Consequently, we decided to explore the effect of depression at two cut-offs of the scale: $\geqslant 6$ ('possible depression') and $\geqslant 7$ ('depression'). History of antidepressant medication use was recorded at baseline and at each follow-up. 


\section{Cognitive function at baseline}

(a) Premorbid IQ was measured using the New Adult Reading Test (NART; Nelson \& O’Connell, 1978).

(b) 'Fluid intelligence' (abstract reasoning, problem-solving) was measured using Raven's Progressive Matrices (Raven, 1940).

(c) Recent memory was assessed with the Paired Associate Learning Test (PALT; Inglis, 1959), which tests the ability to learn and recall cued words. A higher PALT score signifies more recalled words and thus better performance. This was available at baseline and at each wave of data collection.

(d) Attention, concentration and psychomotor function were assessed using the Trail-Making Test (TMT; Reltan, 1959), which is a timed task involving line drawing between numbers on a page. A higher score signifies greater time to complete the task and thus poorer performance.

\section{Cognitive decline}

A summary score, the 'PALT coefficient', reflecting the change in each subject's PALT score over time was calculated by deriving the regression equation for the PALT score against time for each subject (Prince $e t$ al, 1996). A more positive PALT coefficient signifies better performance and therefore less decline in cognition. The TMT was also measured at each wave of data collection. The TMT was converted into a summary measure, the 'TMT coefficient', to reflect the change in each subject's TMT score over time (Prince et al, 1996). A more positive TMT coefficient signifies less improvement with time and thus worse performance.

\section{Cardiovascular risk factors}

Smoking at time of entry to trial was coded as a binary variable (smoker/non-smoker). Non-fasting random serum cholesterol was measured at baseline. Systolic blood pressure was measured by trained research nurses as the sitting blood pressure using a random-zero sphygmomanometer at entry and each follow-up. Body mass index was measured at baseline. Electrocardiogram (ECG) evidence of cardiac ischaemia or arrhythmia was measured using expert analysis of 12-lead electrocardiography.

\section{Statistical analyses}

All analyses were carried out using STATA version 6 (StataCorp, 1999). Univariate associations between the potential explanatory variables and mortality were assessed using classical Mantel-Haenszel methods for rate ratios. Hazard ratios were derived using Cox's regression (Clayton \& Hills, 1996). Assessment of the proportional hazards assumptions was carried out using an Aalen cumulative incidence plot, and a formal test for heterogeneity of the effect of depression over thirds of the follow-up (each with an equal number of deaths). The effect of each continuous and ordered categorical variable on mortality was modelled first to assess whether a factor or linear relationship was the best fit for each variable. Any departure from linearity was formally tested using a likelihood ratio test. The survival probability was plotted for those with and without depression at entry, with all other variables held constant at baseline. Multivariate analyses were carried out using Cox regression to build up a model for the effect of depression on mortality, adding in potential confounding variables one at a time and assessing the contribution of depression within the model following the addition of each confounder using the likelihood ratio test. Potential interactions between depression and any other explanatory variables were first identified by looking for any suggestion of unequal rate ratios using classical Mantel-Haenszel methods and then, if interaction appeared likely, by a formal likelihood ratio test using Cox's regression.

\section{RESULTS}

\section{Descriptive characteristics of the sample}

Table 1 shows the main socio-demographic and clinical characteristics of the sample. Of the 2584 subjects, $42 \%$ were male and $58 \%$ female. The mean age at entry to the trial was 70.3 years. During the follow-up period, 744 subjects $(29 \%)$ died. On the depression scale at baseline, $8.0 \%$ scored $\geqslant 6$ (possible depression) and $4.9 \%$ scored $\geqslant 7$ (depression). The percentages who died in these groups were $32 \%$ and $36 \%$, respectively.

\section{Proportional hazards assumptions}

A formal test for heterogeneity of the effect of depression over thirds of the follow-up (each with an equal number of deaths)
Table I Baseline characteristics of participants $(n=2584)$

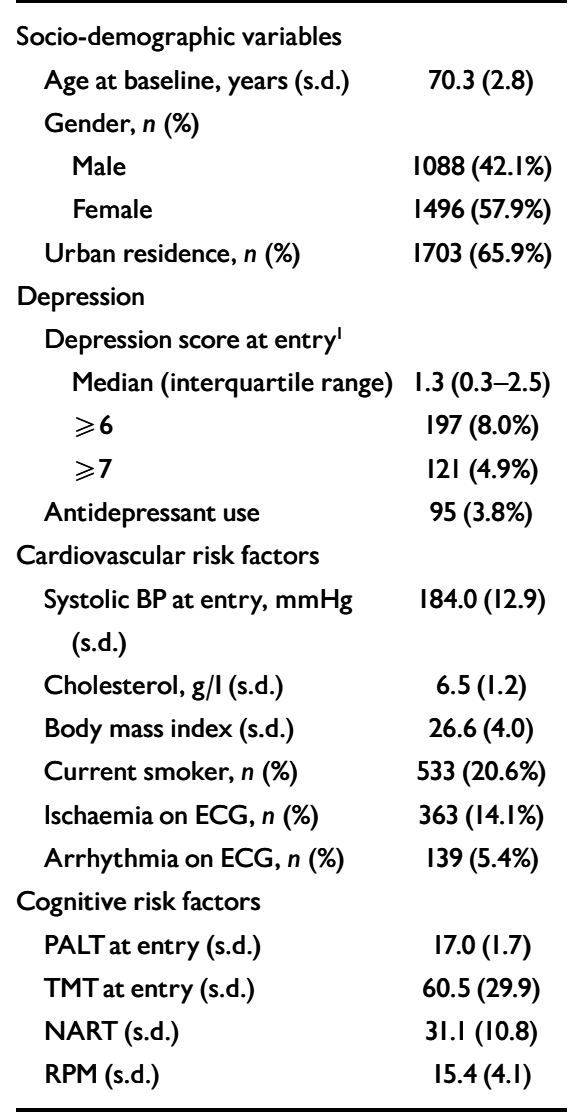

BP, blood pressure; ECG, electrocardiogram; PALT, Paired Associate Learning Test; RPM, Raven's

Progressive Matrices; TMT, Trail-Making Test; NART, New Adult Reading Test.

I. Scored on the 12-item SelfCARE-D depression scale.

showed no statistical evidence of departure from the proportionality assumption $\left(\chi^{2}=0.99, \quad P=0.609\right)$. Thus it was reasonable to use Cox's proportional hazards to model the effect of depression on mortality.

\section{Univariate associations between baseline variables and mortality}

Table 2 describes the association between the main variables and mortality. Depression was only weakly associated when coded as a binary variable $(P=0.07)$. Possible depression was not associated with mortality. Not surprisingly, male gender, older age, smoking, raised systolic blood pressure and ECG ischaemia were all associated with mortality. Lower cholesterol levels were also associated with increased mortality. More died in the beta-blocker group than in the diuretic group $(31 \% v$. $26 \%$ ), with the placebo group intermediate. 
There was no association between mortality and baseline measures of intelligence and cognition. However, decline in cognitive performance over the course of the study was associated with mortality.

\section{Association between depression and mortality controlled for confounders}

Table 3 shows the change in the effect of depression on mortality when various potential confounders are added to the model. Depression (i.e. scoring $\geqslant 7$ on the depression scale) becomes more powerfully associated once gender is controlled for. This reflects the higher prevalence of depression in females and the higher death rate in males. There was some confounding by cardiovascular risk factors and by urban residence. The full model, which controlled for gender, age, cardiovascular risk factors, cognitive decline and urban residence, showed that depression was still modestly associated with mortality. The effect of depression scored as a continuous variable was similar. Assuming an overall prevalence of major depression of $5 \%$ and a hazard ratio of 1.43 , we calculate the population-attributable risk percentage of depression on mortality to be $2.1 \%$.

Possible depression (i.e. scoring $\geqslant 6$ on depression scale) had a modest effect on mortality after controlling for gender (hazard ratio $=1.33 ; 95 \%$ CI $1.12-1.72$, $P=0.041$ ). The relationship was confounded by cardiovascular risk factors and by urban residence. After adjustment for all these factors, the hazard ratio was 1.22 (95\% CI 0.92-1.63, $P=0.18$ ).

\section{Interaction between depression and gender and depression and age}

We detected an interaction term $\left(\chi^{2}=3.05\right.$, 1 d.f., $P=0.081$ ) between depression and gender. Males with depression had considerably increased risk of dying (hazard ratio $=2.12$, 95\% CI 1.28-3.52) even after adjustment for cardiovascular and cognitive risk factors and for urban residence. In females the risk was not significantly increased (hazard ratio $=1.14, \quad 95 \%$ CI $0.75-1.73)$. There was also a significant interaction term between age and depression. Younger people with depression were at increased risk (Table 4), but depression in those aged over 70 years at baseline did not appear to be associated with mortality.
Table 2 Univariate associations with mortality

\begin{tabular}{|c|c|c|c|}
\hline & $\begin{array}{c}\text { Hazard ratio } \\
\qquad(95 \% \mathrm{Cl})\end{array}$ & $\begin{array}{l}\text { Likelihood ratio } \\
\text { test } \chi^{2} \text { (d.f.) }\end{array}$ & $P$ \\
\hline \multicolumn{4}{|l|}{ Demographics } \\
\hline Female gender & $0.52(0.45-0.60)$ & $80.01(1)$ & $<0.001$ \\
\hline Urban residence & $1.17(1.0 \mathrm{I}-1.36)$ & $4.10(1)$ & 0.043 \\
\hline Age at baseline' & I.2I (I.II-I.32) & $17.98(I)$ & $<0.001$ \\
\hline \multicolumn{4}{|l|}{ Cardiovascular risk factors } \\
\hline Smoker at entry & $1.82(1.56-2.14)$ & $51.32(1)$ & $<0.001$ \\
\hline Systolic BP at entry ${ }^{2}$ & $1.00(1.00-1.01)$ & $\mathrm{I} .55(\mathrm{I})$ & 0.210 \\
\hline Mean BP over trial' & I.34 (I.23-I.47) & $42.18(I)$ & $<0.001$ \\
\hline Cholesterol at entry' & $0.80(0.73-0.87)$ & $25.55(I)$ & $<0.001$ \\
\hline ECG ischaemia at entry & $1.37(1.13-1.66)$ & $9.89(\mathrm{I})$ & 0.002 \\
\hline ECG arrhythmia at entry & $0.82(0.61-I .10)$ & $\mathrm{I} .73(\mathrm{I})$ & 0.190 \\
\hline Body mass index' & $0.93(0.85-1.01)$ & $2.62(\mathrm{I})$ & 0.110 \\
\hline \multicolumn{4}{|l|}{ Treatment group } \\
\hline Diuretic $v$. rest & $0.86(0.72-1.02)$ & $3.2(I)$ & 0.078 \\
\hline Beta-blocker v. rest & I.II (0.94-I.3I) & $\mathrm{I} .59(\mathrm{I})$ & 0.200 \\
\hline \multicolumn{4}{|l|}{ Depression } \\
\hline \multicolumn{4}{|l|}{ Depression score } \\
\hline$\geqslant 6$ out of 12 & I.I4 (0.88-I.47) & $0.90(\mathrm{I})$ & 0.340 \\
\hline$\geqslant 7$ out of 12 & I.32 (0.97-I.79) & $2.96(\mathrm{I})$ & 0.073 \\
\hline Antidepressant use at baseline & $1.07(0.72-1.58)$ & $0.12(I)$ & 0.730 \\
\hline \multicolumn{4}{|l|}{ Cognitive risk factors } \\
\hline NART score' & $0.94(0.86-1.02)$ & $2.02(I)$ & 0.160 \\
\hline Raven's Progressive Matrices score & $0.97(0.88-1.06)$ & $0.40(\mathrm{I})$ & 0.530 \\
\hline PALT at entry' & $0.95(0.87-1.03)$ & $\mathrm{I} .26(\mathrm{I})$ & 0.260 \\
\hline \multicolumn{4}{|l|}{ PALT coefficient } \\
\hline Mid v. most decline & $0.79(0.66-0.94)$ & & \\
\hline Least $v$. most decline & 0.91 (0.77-I.09) & $7.35(2)$ & 0.025 \\
\hline $\mathrm{TMT}^{\prime}$ at entry & $1.05(0.96-1.14)$ & $0.99(I)$ & 0.320 \\
\hline \multicolumn{4}{|l|}{ TMT coefficient } \\
\hline Mid v. most improved & $0.79(0.78-I .12)$ & & \\
\hline Least $v$. most improved & $1.25(1.06-1.49)$ & $12.40(2)$ & 0.002 \\
\hline
\end{tabular}

BP, blood pressure; ECG, electrocardiogram; NART, New Adult Reading Test; PALT, Paired Associate Learning Test; TMT, Trail-Making Test.

I. Linear increase in thirds.

2. Linear increase in quarters.

Table 3 Cox's regression showing the association between depression at trial entry and mortality, adjusting for confounding variables

\begin{tabular}{|c|c|c|c|}
\hline $\begin{array}{l}\text { Depression (scoring } \geqslant 7 \\
\text { on depression scale) }\end{array}$ & $\begin{array}{l}\text { Hazard ratio for } \\
\text { mortality }(95 \% \mathrm{Cl})\end{array}$ & $\begin{array}{c}\text { Likelihood ratio } \\
\text { test } \chi^{2}\end{array}$ & $P$ value \\
\hline Depression (univariate) & I.32 (0.97-I.79) & 2.96 & 0.086 \\
\hline Depression adjusted for gender & $1.55(I .14-2.11)$ & 6.96 & 0.008 \\
\hline Depression adjusted for variable I & $1.56(1.15-2.13)$ & 7.18 & 0.007 \\
\hline Depression adjusted for I, 2 & $1.50(1.10-2.05)$ & 5.99 & 0.014 \\
\hline Depression adjusted for I, 2, 3 & I.5I (I.II-2.05) & 6.05 & 0.014 \\
\hline Depression adjusted for I, 2, 3, 4 & $\mathrm{I} .48(\mathrm{I} .08-2.0 \mathrm{I})$ & 5.47 & 0.019 \\
\hline Depression adjusted for $I, 2,3,4,5$ & $1.43(1.03-1.98)$ & 4.21 & 0.040 \\
\hline
\end{tabular}

Confounding variables: I, gender and baseline antidepressant use; 2, cardiovascular risk factors (i.e. cholesterol, smoking, mean blood pressure, electrocardiogram (ECG) ischaemia, ECG arrhythmia, randomised antihypertensive treatment); 3, IQ (New Adult Reading Test and Raven's Progressive Matrices) and cognitive decline (Paired Associated Learning Test coefficient, Trail-Making Test coefficient); 4, age; 5, urban residence. 
Table 4 The interaction between depression on mortality, by age group'

\begin{tabular}{lccc}
\hline Depression (scoring $\geqslant 7$ on depression scale) & $\begin{array}{c}\text { Hazard ratio for mortality } \\
(95 \% \mathrm{Cl})\end{array}$ & Wald test & $P$ \\
\hline $\begin{array}{c}\text { Effect of depression in youngest third } \\
\text { (aged 65-69 years at study entry) }\end{array}$ & $2.26(1.34-3.80)$ & 3.06 & 0.004 \\
$\begin{array}{c}\text { Effect of depression in middle third } \\
\quad \text { (aged 69-7I years at study entry) }\end{array}$ & $1.27(0.69-2.36)$ & 0.77 & 0.44 \\
$\begin{array}{c}\text { Effect of depression in oldest third } \\
(\text { aged } 72-75 \text { years at study entry) }\end{array}$ & $0.95(0.54-1.68)$ & -0.18 & 0.89 \\
\hline
\end{tabular}

I. Interaction parameter: mid-age compared with youngest age $=0.57(0.25-1.27)$, Wald $z=-1.401, P=0.161$; oldest age compared with youngest age $=0.43(0.19-0.91)$, Wald $z=-2.201, P=0.028$. Likelihood ratio test for overall interaction $\chi^{2}(2$ d.f. $)=4.93, P=0.085$.

2. Adjusted for cholesterol, smoking, mean blood pressure, electrocardiogram ischaemia, randomised antihypertensive treatment, cognitive decline, gender and urban residence.

\section{Antidepressant use and mortality}

There were no significant associations between antidepressant use at trial entry and mortality. The hazard ratio for antidepressant use at entry, adjusted for gender, cardiovascular risk factors, age, urban residence and depression score, was 1.01 (95\% CI $0.65-1.55, P=0.99)$. The hazard ratio for antidepressant use during the trial (linear increase in quarters), adjusted for gender, cardiovascular risk factors, age, urban residence and depression score, was 1.06 (95\% CI $0.99-1.13, P=0.084)$.

\section{DISCUSSION}

The long follow-up of a group at high risk of mortality (elderly hypertensive) gave us sufficient power to detect a modest association between mortality and depression. The association is only present after controlling for gender, and appears to be most powerfully present in males and younger subjects. The association was not explained by most conventional cardiovascular risk factors or by cognitive decline. Antidepressant use was not associated with mortality.

\section{Study limitations}

The study restricted the sample to a relatively healthy group. Thus, it is unlikely that the association is due to the presence of physical illness among those rated as depressed at baseline. However, there were potential methodological flaws in the design of this study. First, we concentrated on a high-risk group of elderly subjects with hypertension who had agreed to participate in a randomised trial; therefore the results may be of limited generalisability and may not apply to younger and normotensive populations. Second, although we were able to control for a wider range of confounders than most previous studies, smoking was only coded as a binary variable. This overaggregation might lead to residual confounding. However, this may not be of major concern because controlling for cardiovascular risk factors only reduced the hazard ratio from 1.56 to 1.50 . One would expect a much more dramatic decrease in the hazard ratio when controlling for this confounder if residual confounding was capable of explaining the association. Third, our measure of depression was a questionnaire. It is possible that this is less valid than clinical interviews, although the SelfCARE-D has been shown to have acceptable validity and reliability in this age group (Bird et al, 1987; Upadhyaya \& Stanley, 1997). If there was misclassification of cases it is most likely that this would be random misclassification, and thus lead to underestimation of effect size.

\section{Major versus minor depression}

Our finding is consistent with recent findings from the longitudinal Amsterdam study reporting a greater effect on mortality for major depression than for minor depression (Penninx et al, 1999). Given the finding by Blanchard et al (1994), using a comprehensive structured clinical assessment for depression in the elderly (GMS AGECAT; Copeland et al, 1986), that the mean score for cases of depression in an elderly community sample was $\geqslant 7$ on the SelfCARE-D, it is likely that the $\geqslant 7$ cutoff provides greater specificity and/or indicates greater severity than the $\geqslant 6$ cutoff. The effect of depression that we found appeared greater and was more statistically significant at the higher cut-off of the SelfCARE-D (i.e. at $\geqslant 7$ out of 12 compared with $\geqslant 6$ ). At the $\geqslant 6$ cut-off $8 \%$ were depressed, and at the $\geqslant 7$ cut-off $4.9 \%$ were depressed. If $\geqslant 6$ represents possible/ minor depression, this prevalence is somewhat lower than reported from community studies (e.g. 12.8\%; Penninx et al, 1999). Given that our population is from a randomised trial, this probably reflects the healthy volunteer effect.

\section{Male gender and mortality from depression}

The increased risk associated with depression was present in males (but not females) and in the younger age group in this sample. The interaction terms that we detected were of only borderline statistical significance $(0.05<P<0.1)$ and may represent type I error. However, our findings are consistent with recent papers suggesting that the effect of depression on mortality is greater in men than in women (Mallon et al, 2000). Furthermore, Pennix et al (1999: p. 63) reported that although major depression predicted mortality in males and females, minor depression predicted mortality only in males. We are not aware of any research to date that has detected age differences in the effect of depression on mortality, so this finding requires replication.

\section{Mediating factors}

Given the increasing evidence for the association between depression and cardiac mortality (Penninx et al, 2001) and the association between male gender and cardiovascular disease, it could be that the effect of depression is partly explained by cardiovascular and/or cerebrovascular disease, which has not been dealt with in the analysis. Although suicide may be another explanation for the excess mortality in males with depression, this is unlikely because it is a rare cause of death in community samples (Penninx et al, 1999). It would be valuable to carry out further analysis in our sample to look at causespecific mortality.

The literature that has previously linked depression and mortality has proposed two main mechanisms. The first relates to lifestyle, namely that depression may be a more distal risk factor for a number of risk factors that increase morbidity and mortality from cardiovascular and other physical disease. For example, people with depression may 
smoke more, exercise less, adhere less to treatment and drink more alcohol (Allgulander, 1994; Blanchard et al, 1994). We have been able to study markers for cardiovascular disease, including ECG ischaemia and smoking. Although these sorts of variable have been dealt with as confounders in this analysis, they are, strictly speaking, intermediary factors on the same causal pathway. Our analyses suggest that the association is unlikely to be entirely explained by these risk factors, although we were unable to control effectively for exercise or alcohol intake.

The second mechanism involves a group of risk factors that relate to possible biological change occurring in depression. Depression is known to be associated with increased cortisol, increased platelet coagulability and changes in heart rate variability, perhaps indicating a cardiovascular system more prone to arrhythmias (Dalack \& Roose, 1990; Mikuni et al, 1992). These findings are predominantly derived from small clinical samples of patients with severe depression. The next step will be to explore such associations in large epidemiologically based samples. We were able to adjust for ECG evidence of arrhythmia and hence this does not appear to have mediated the association between depression and mortality.

\section{Future research}

In terms of public health implications, depression is a common exposure. Most people with depression in the community do not receive any specific treatment for depressive symptoms (Schoevers et al, 2000). Two early studies suggest that treatment of depression may lower mortality, especially in men (Avery \& Winokur, 1976; Craig \& Lin, 1981). The findings here, together with the previous literature, suggest the need to investigate the effect on mortality of vigorous treatment of confirmed depression.

\section{ACKNOWLEDGEMENTS}

The authors thank the Medical Research Council (MRC) Working Party (permission to use the data), Mr Pat Brennan (computing/raw data sets), Mrs Irene Godson (data entry), Professor A. Mann (psychiatric substudy development) and Professor T. Meade, Mrs W. Browne, Dr J. Connolly and the MRC Research nurses and doctors from the MRC General Practice Research Framework (collecting, collating and providing the original study data). The study was begun when M.A. was studying for the

\section{CLINICAL IMPLICATIONS}

Major depression is associated with increased mortality in older people, but the effect size is small (population-attributable risk percentage around $2 \%$ ).

- During episodes of depression males are at greater risk of mortality than females.

- Previous antidepressant use is not associated with an increased mortality risk.

\section{LIMITATIONS}

- The study was restricted to people aged over 65 years with moderate hypertension at baseline.

Depression status relied on the SelfCARE-D score, which has limited reliability and validity.

We did not have data on the causes of death.

MELANIE ABAS, MRCPsych, Division of Psychiatry, University of Auckland, New Zealand; MATTHEW HOTOPF, PhD, Department of Psychological Medicine, Guy's, King's and St Thomas' School of Medicine and Institute of Psychiatry, London, UK; MARTIN PRINCE, MD, Section of Epidemiology, Institute of Psychiatry, London, UK

Correspondence: Dr Melanie Abas, Research and Audit in Mental Health ServicesTeam, Tiaho Mai, Middlemore Hospital, South Auckland, New Zealand. E-mail: m.abas@auckland.ac.nz

(First received 3 December 200I, final revision 23 April 2002, accepted 23 April 2002)

MSc in Epidemiology at the London School of Hygiene and Tropical Medicine, supported by the Thames Postgraduate Dean (Flexible Training) and an Eli Lilly Educational Award.

\section{REFERENCES}

Allgulander, C. (1994) Suicide and mortality patterns in anxiety neurosis and depressive neurosis. Archives of General Psychiatry, 5I, 708-712.

Avery, D. \& Winokur, G. (1976) Mortality in depressed patients treated with electroconvulsive therapy and antidepressants. Archives of General Psychiatry, 33, 1029-1037.

Banerjee, S., Shamash, K., MacDonald, A. J., et a (1998) The use of the SelfCARE (D) as a screening too for depression in the clients of local authority home care services - a preliminary study. International journal of Geriatric Psychiatry, I3, 695-699.

Bird, A.S., MacDonald, A.J., Mann, A.H., et al (1987) Preliminary experience with the SelfCARE (D): a selfrating depression questionnaire for use in elderly, noninstitutionalized subjects. International journal of Geriatric Psychiatry, 2, 31-38.

\section{Blanchard, M. R., Waterreus, A. \& Mann, A. H.}

(1994) The nature of depression among older people in inner London, and the contact with primary care. British Journal of Psychiatry, 164, 396-402.

Clayton, D. \& Hills, M. (1996) Statistical Models in Epidemiology. Oxford: Oxford University Press.
Copeland, J. R., Dewey, M. E. \& Griffiths-Jones, H. M. (1986) A computerized psychiatric diagnostic system and case nomenclature for elderly subjects: GMS and AGECAT. Psychological Medicine, 16, 89-99.

Craig, T. J. \& Lin, S. P. (198I) Mortality among elderly psychiatric patients: basis for preventive intervention. Journal of the American Geriatrics Society, 29, I8I-185.

Dalack, G.W. \& Roose, S. P. (1990) Perspectives on the relationship between cardiovascular disease and affective disorder. Journal of Clinical Psychiatry, 5I, 4-9.

Inglis, J. (1959) A paired-associate learning test for use with elderly psychiatric patients. Journal of Mental Science, 105, 440-443.

Ismail, K., Sloggett, A. \& De Stavola, B. (2000) Do common mental disorders increase cigarette smoking? Results from five waves of a population-based panel cohort study. American Journal of Epidemiology, 152. 65I-657.

Mallon, L., Broman, J. E. \& Hetta, J. (2000) Relationship between insomnia, depression, and mortality: a 12-year follow-up of older adults in the community. International Psychogeriatrics, 12, 295-306.

Mikuni, M., Kagaya, A., Takahashi, K., et al (1992) Serotonin but not norepinephrine-induced calcium mobilization of platelets is enhanced in affective disorders. Psychopharmacology, 106, 31I-314.

MRC Working Party (1992) Medical Research Council trial of treatment of hypertension in older adults: principal results. BMJ, 304, 405-4I2. 
Nelson, H. E. \& O'Connell, A. (1978) Dementia: the estimation of premorbid intelligence levels using the New Adult Reading Test. Cortex, 14, 234-244.

Penninx, B.W., Geerlings, S.W., Deeg, D. J., et al (1999) Minor and major depression and the risk of death in older persons. Archives of General Psychiatry, 56 889-895.

_ , Beekman, A. T., Honig, A., et al (200I) Depression and cardiac mortality: results from a community-based longitudinal study. Archives of General Psychiatry, 58, 221-227.

Prince, M. J., Bird, A. S., Blizard, R. A., et al (1996) Is the cognitive function of older patients affected by antihypertensive treatment? Results from 54 months of the Medical Research Council's trial of hypertension in older adults. BMJ, 312, 80I-805.
Raven, J. C. (1940) Matrix tests. Mental Health, I, $10-18$

Reltan, R. M. (1959) Validity of the trail making test as an indicator of organic brain damage. Perceptual Motor Skills, 8, 27I-276.

Saz, P. \& Dewey, M. E. (200I) Depression, depressive symptoms and mortality in persons aged 65 and over living in the community: a systematic review of the literature. International Journal of Geriatric Psychiatry, 16, 622-630.

Schoevers, R. A., Beekman, A. T., van Tilburg, W., et al (2000) Association of depression and gender with mortality in old age. Results from the Amsterdam Study of the Elderly (AMSTEL). British Journal of Psychiatry, 177 336-342.
StataCorp (1999) Stata Statistical Software (Version Release 6.0). College Station, TX: Stata Corporation.

Upadhyaya, A. K. \& Stanley, I. (1997) Detection of depression in primary care: comparison of two selfadministered scales. International Journal of Geriatric Psychiatry, 12, 35-37.

Witthaus, E., Ott, A., Barendregt, J. J. et al (1999) Burden of mortality and morbidity from dementia. Alzheimer Disease and Associated Disorders, I3, |76-181.

Wulsin, L. R., Vaillant, G. E. \& Wells, V. E. (1999) A systematic review of the mortality of depression. Psychosomatic Medicine, 6I, 6-17. 